\title{
Prevalence of asymptomatic malaria, submicroscopic parasitaemia and anaemia in Korogwe District, north-eastern Tanzania
}

Paul M. Hayuma ${ }^{1,2^{*}}$, Christian W. Wang ${ }^{3,4}$, Edwin Liheluka', Vito Baraka ${ }^{1}$, Rashid A. Madebe ${ }^{1}$, Daniel T. R. Minja', Gerald Misinzo ${ }^{2,5}$, Michael Alifrangis ${ }^{3,4}$ and John P. A. Lusingu ${ }^{1}$

\begin{abstract}
Background: Asymptomatic malaria infections largely remain undetected and act as a reservoir for continuous transmission. The study assessed the prevalence of submicroscopic asymptomatic malaria infections and anaemia in two rural low (300 m above sea level) and highland (700 m asl) settings of Korogwe District north-eastern Tanzania.

Methods: A cross-sectional malariometric survey involving individuals aged 0-19 years was conducted in June 2018 in the two rural villages. Venous blood was collected from eligible study participants for estimation of haemoglobin level, detection of malaria by rapid diagnostic test (RDT), quantification of malaria parasitaemia by microscopy, as well as dried blood spot (DBS) for determining submicroscopic infections by PCR targeting the small subunit of the ribosomal ribonucleic acid (ssrRNA) of human Plasmodium.
\end{abstract}

Results: Out of 565 individuals tested, 211 (37.3\%) were malaria positive based on RDT, whereas only 81 (14.3\%) were positive by microscopy. There was no significant difference in the prevalence between the highland and the lowland village, $p=0.19$ and $p=0.78$ microscopy and RDT, respectively. Three out of 206 (1.5\%) RDT/microscopy negative samples were P. falciparum positive by PCR. Of the 211 RDT and 81 microscopy positive, 130 (61.6\%) and 33 (40.7\%), respectively, were defined as being asymptomatic. Of the 565 individuals, 135 (23.9\%) were anaemic (haemoglobin $<11 \mathrm{~g} / \mathrm{dL}$ ) out of which $5.2 \%$ were severely anaemic. The risk of being anaemic was significantly higher among individuals with asymptomatic malaria as compared to those without malaria as confirmed by RDT (AOR $=2.06$ ( $95 \%$ $\mathrm{Cl} 1.32-3.20$ ) while based on microscopic results there was no significant differences observed ( $\mathrm{AOR}=2.09,95 \% \mathrm{Cl}$ 0.98-4.47). Age and altitude had no effect on the risk of anaemia even after adjusting for asymptomatic malaria.

Conclusions: Asymptomatic malaria is associated with an increased risk of having anaemia in the study communities. The findings highlight the need for targeted interventions focusing on asymptomatic infections which is an important risks factor for anaemia in the community and act as a source of continued transmission of malaria in the study area.

Keywords: Plasmodium falciparum, Asymptomatic infection, Anaemia, Submicroscopic parasitaemia, North-eastern Tanzania

*Correspondence: martineson2016@gmail.com

${ }^{1}$ National Institute for Medical Research, Tanga Research Centre, P. O. Box 5004, Tanga, Tanzania

Full list of author information is available at the end of the article

\section{Background}

Malaria claims lives of more than 400,000 people annually [1]. Reportedly, there has been a global reduction in malaria cases from 238 to 229 million between 2000 and 2019, but the sub-Saharan Africa (SSA) still experiences original author(s) and the source, provide a link to the Creative Commons licence, and indicate if changes were made. The images or other third party material in this article are included in the article's Creative Commons licence, unless indicated otherwise in a credit line to the material. If material is not included in the article's Creative Commons licence and your intended use is not permitted by statutory regulation or exceeds the permitted use, you will need to obtain permission directly from the copyright holder. To view a copy of this licence, visit http://creativecommons.org/licenses/by/4.0/. The Creative Commons Public Domain Dedication waiver (http://creativeco mmons.org/publicdomain/zero/1.0/) applies to the data made available in this article, unless otherwise stated in a credit line to the data. 
an unprecedented disease burden attributable to malaria [1]. The existing malaria control tools including the deployment of effective anti-malarial drugs, such as artemisinin-based combination therapy (ACT) has substantially contributed in curtailing transmission through its gametocytocidal effect and hence influenced the observed reduction in malaria related morbidities and mortalities [2].

In Tanzania, in addition to ACT use, successful implementation of other malaria preventive strategies including wide use of long-lasting insecticidal nets could most likely explain the observed decline in malaria prevalence in the general population from 18 to $7 \%$ between 2008 and 2017 [3]. Various epidemiological studies conducted in north-eastern Tanzania have reported a significant decline in malaria between 2003 and 2012 [4-7] followed by a temporal increase in 2016 and 2017 [4, 5].

In malarial endemic settings, repeated infections cause a partial non-sterilizing immunity to malaria resulting into asymptomatic and often, chronic infections that are rarely detected and may end up not being treated [8]. Many of these asymptomatic malaria carriers maintain low level parasitaemia and gametocytaemia which may play a significant role in transmission [9]. Moreover, even low level malaria parasitaemia is associated with an increased risk of anaemia $[10,11]$ and may impair cognitive development [12].

Detection of malaria parasites by microscopy (gold standard) or rapid diagnostic test (RDT) are challenged by a number of factors including limited availability of expert microscopists, low detection rates, especially when the parasitaemia is too low and high level false positive rates with RDT due to circulating parasite antigens [13]. The use of molecular techniques that detect parasite DNA offers an important research tool that can reveal submicroscopic parasitaemia undetectable by these conventional malaria diagnostic tools [8].

Despite the realized success in malaria control from various control interventions that have resulted into a significant decline in malaria related morbidity and mortality in north-eastern Tanzania [6-8], there is still paucity of data on the prevalence of asymptomatic malaria infections and its association with anaemia in this malaria endemic setting. The present study aimed to determine the prevalence of asymptomatic, submicroscopic malaria infections and anaemia in two rural communities of Kwamasimba (highland village) and Mkokola (lowland village,) in Korogwe District in north-eastern Tanzania.

\section{Methods}

\section{Study sites}

This cross-sectional study was conducted in two villages of Korogwe District, namely Mkokola (a village in a lowland area, $300 \mathrm{~m}$ asl) and Kwamasimba (a highland village, $700 \mathrm{~m}$ asl) in north-eastern Tanzania. The prevalence of $P$. falciparum malaria parasitaemia in the lowland village has been on decline from $78 \%$ to 2003 to $13 \%$ in 2008, whereas in the highland village, the prevalence decreased from 25 to $3 \%$ in the same period [7]. Subsistence farming and informal petty trading are the main income generating activities in this rural setting. The data presented here is of over 10 years as this data come from a continuous malaria project in the same study area from 2003 to date. The data published in public domain of is of 2003 to 2008. The available unpublished data of 2009 to 2018 has different trends of malaria prevalence in the study area, this data will be published later.

\section{Study design and population}

This study collected data from a cross-sectional malariometric survey as part of the main study entitled "Malaria Research Capacity building for field clinical trial in Tanzania" (MaReCa Project-TMA 2015 SF-998) in two study villages. Analysed data included malaria epidemiological data collected in specially designed questionnaires (clinical, demographic and socioeconomic data) from individuals aged 0-19 years. Laboratory data were obtained from RDT, haemoglobin measurements by HemoCue machines, and typing for submicroscopic parasitaemia by PCR from parasite DNA extracted from dried blood spots (DBS). The prevalence of submicroscopic infection was determined from DBS that were selected based microscopic negative blood smears and were as well RDT negative. The DBS $(n=206,43 \%)$ were selected from RDT and blood smears negatives to determine the prevalence of submicroscopic infections.

\section{Malariometric survey}

The cross-sectional malariometric survey was conducted in June 2018 soon after cessation of the long rainy season with concomitant peak malaria transmission. Sociodemographic information including age, gender and place of residence were recorded using standardized questionnaires. Axillary body temperature was measured using digital thermometers (Omron, Turkey). Symptoms related to malaria such as fever, vomiting, convulsion, headache, loss of appetite and general body malaise were also recorded. Also study participants were asked if they have been taken any anti-malarial treatment within 14 days prior to enrollment into the study. Venous blood was collected in ethylenediaminetetraacetic acid (EDTA) vacutainers and blood remaining in the butterfly tube was used to prepare DBS on Whatman filter paper (Whatman \#3, WVR, Søborg, Denmark), dried at room temperature and stored in silica gel). Malaria was tested by using thick and thin blood smears microscopy, as well as RDT (Care 
Start ${ }^{\mathrm{TM}}$, Somerset, NJ USA) with both histidine rich protein 2 (HRP-2) and parasite lactate dehydrogenase (pLDH) following the manufacturer's instructions. Haemoglobin $(\mathrm{Hb})$ measurement was done in the field using HemoCue 301 machine (Angelholm, Sweden). All participants with uncomplicated malaria based on RDT and other clinical algorithms as per clinician discretions were treated using artemether-lumefantrine (AL) according to the existing Tanzanian malaria treatment guidelines [14]. However, for severely sick patients referrals to the nearby health facility were made as appropriate.

\section{Microscopic examination of thick and thin blood smears}

Thin blood smears for the detection and quantification of malaria parasites were fixed with absolute methanol. Both thick and thin smears were then stained with $10 \%$ Giemsa for $30 \mathrm{~min}$, gently washed with tap water, air dried and examined at $100 \times$ objectives using a binocular microscope (CX23 Olympus Corporation, Tokyo, Japan). Asexual parasites were counted against 200 leukocytes and sexual parasite counts were done against 500 leukocytes. If the asexual parasite counts were less than 10 , reading was done per 500 leukocytes. All slides were read twice by two independent, certified microscopists and results from the two readings with a difference of less than $50 \%$ were considered definitive. Smears with discordant results were re-examined by a third reader blinded to the first and second readings. Results from the two independent readers that were in agreement were considered final. A slide was declared negative if no parasites were seen after scanning 200 high power fields. All microscopists who read the blood slides participated in proficient microscopic examination and were certified by the National Institute for Communicable Diseases (NICD), South Africa.

\section{Genomic DNA extraction and molecular detection of malaria parasites by PCR}

Malaria parasite DNA was extracted from randomly selected DBS that were collected from RDT negative individuals and confirmed to be negative by microscopy. The DNA was extracted using QIAamp DNA Blood mini kit (Qiagen GmbH, Hilden, Germany), as per manufacturer's instructions. After extraction, the eluted DNA was dispensed into $1.5 \mathrm{~mL}$ Eppendorf tubes and stored at $-20{ }^{\circ} \mathrm{C}$. One microlitre of parasite genomic DNA was amplified using a thermocycler (T Professional, Thermocycler Biometra GmbH, Saxony, Germany). Detection submicroscopic infections of Plasmodium species was done by Plasmodium genus and species-specific PCR targeting the small subunit of the ribosomal ribonucleic acid (ssrRNA) genes of human Plasmodium using conditions as previously described [15]. Briefly, the master-mix reagents per sample comprised of; $1 \mu \mathrm{L}$ of primer mix (forward and reverse primers $0.25 \mathrm{nM}$ ), $10 \mu \mathrm{L}$ of Tempase and $8 \mu \mathrm{L}$ of PCR quality water. Targeted species were Plasmodium falciparum, Plasmodium malariae and Plasmodium ovale. Plasmodium falciparum forward and reverse primers $5^{\prime}$-TTA AAC TGG TTT GGG AAA ACC AAA TAT ATT-3' 5' $^{\prime}$-ACA CAA TGA ACT CAA TCA TGA CTA CCC GTC-3', respectively. Plasmodium malariae forward and reverse primers $5^{\prime}$ AAA ATT CCA TGC ATA AAA TTA TAC AAA-3' ${ }^{\prime}$, ${ }^{\prime}$-ATC TCT TTT GCT ATT TTT TAG TAT TGG AGA-3', respectively. Plasmodium ovale forward and reverse primers $5^{\prime}$-ATC TCT TTT GCT ATT TTT TAG TAT TGG AGA-3', GGA AAA GGA CAT TAA TTG TAT CCT AGTG-3', respectively.

For each species targeted positive and negative controls were run together with the extracted DNA samples. To ensure quality of the PCR, positive and negative controls were included in each set of PCR run. As positive control for P. falciparum 3D7 strain and DNA extracted from DBS collected from microscopy confirmed $P$. falciparum positive cases whereas for $P$. malariae and $P$. ovale parasite DNA was extracted from microscopy confirmed $P$. malariae and $P$. ovale cases. Negative controls used was ultrapure water. Ethidium bromide stained $2 \%$ agarose gel was used to resolve amplicons under Ultra Violet-BioDoc Analyzer (Biometra GmbH, Saxony, Germany).

\section{Data management and analysis}

Data were entered and validated using a double entry system in Microsoft access (Version 2007). Data analysis was done using STATA statistical software package version 13.1 (Stata Corp College Station, Texas USA). Proportions between groups were compared using $X^{2}$-test. Univariate and multivariate logistic regression analyses were used to assess the associations between anaemia with asymptomatic malaria, gender, altitude and age. Effects sizes were presented using odds ratio (OR) with $95 \%$ confidence intervals (CI). P-value of $<0.05$ was considered statistically significant.

\section{Case definitions}

The study enrolled individuals aged $0-19$ years with permanent residence in the study area. These individuals ( $<19$ years) willingly consented to participate or their parents/guardians gave an informed consent for their participation. Individuals with positive RDT/microscopy and temperature above $37.5{ }^{\circ} \mathrm{C}$ and as well with signs of malaria, such as vomiting, convulsion, general body malaise, headache and/or loss of appetite, were considered symptomatic. Asymptomatic malaria individuals were defined as those individuals who had axillary body temperature below $37.5{ }^{\circ} \mathrm{C}$, no history of fever for 
the past $48 \mathrm{~h}$ and were malaria positive by RDT, microscopy or PCR [16]. Submicroscopic malaria carriers were defined as individuals who were malaria positive based on PCR but were both microscopy and RDT negative. Anaemia was defined according to the World Health Organization (WHO) age-adjusted cut-off for haemoglobin (Hb) levels [17], whereby a Hb level $<7.0 \mathrm{~g} / \mathrm{dL}$ was regarded as severe anaemia, between 7.0 and $9.9 \mathrm{~g} / \mathrm{dL}$ as moderate anaemia, between 10.0 and $10.9 \mathrm{~g} / \mathrm{dL}$ as mild anaemia and $\mathrm{Hb}>11 \mathrm{~g} / \mathrm{dL}$ as non-anaemic.

\section{Results}

\section{Baseline characteristics of the study participants}

A total of 565 study participants were enrolled of whom 280 were from the highland village and the remaining $(n=285)$ were from the lowland village. The majority of the participants were children aged below 5 years

Table 1 Demographic information of study participants from Kwamasimba (highland) and Mkokola (lowland) villages, Korogwe District, north-eastern Tanzania

\begin{tabular}{lll}
\hline Variable & $\begin{array}{l}\text { Number of } \\
\text { participants }\end{array}$ & Percentage (\%) \\
\hline Strata & & \\
$\quad$ Highland village, Kwamasimba & 280 & 49.6 \\
$\quad$ Lowland village, Mkokola & 285 & 50.4 \\
Sex & & \\
Male & 275 & 48.8 \\
Female & 290 & 51.2 \\
Age groups (years) & & \\
0-4 & 237 & 42.0 \\
$5-9$ & 145 & 25.6 \\
10-14 & 118 & 20.9 \\
$15-19$ & 65 & 11.5 \\
\hline
\end{tabular}

(42.0\%), followed by 5-9 years $(25.7 \%)$ and the least were those aged $17-19$ years $(11.5 \%)$, Table 1 .

\section{Prevalence of asymptomatic malaria and submicroscopic parasitaemia in highland and lowland villages}

The overall malaria point prevalence in the two villages was $37.3 \%(211 / 565)$ as determined by RDT, but with almost 2.5 -fold drop in prevalence $14.3 \%$ (81/565) as revealed by microscopy (Table 2 ). Upon stratification by altitude, the highland village showed an overall malaria point prevalence of $38.2 \%(107 / 280)$ and $18.6 \%(52 / 280)$ based on RDT and microscopy, respectively. On the other hand, in the lowland village the overall malaria point prevalence stood at $36.5 \%(104 / 285)$ based on RDT and $10.2 \%(29 / 285)$ by microscopy (Table 2$)$. Among the 211 positive based on RDT results $38.4 \%$ (81/211) were symptomatic whereas based on microscopy results out 81 positive $59.3 \%(48 / 81)$ where symptomatic. Of the 211 RDT and 81 microscopy positive, 61.6\% (95\% CI 54.9-68.2\%, (130/211) and 41.0\% (95\% CI 28.6-50.4\%, (33/81) were defined as asymptomatic malaria infections, respectively. Again upon stratification by location, $63 / 280(22.5 \%)$ and $20 / 280(7.1 \%)$ in the highland villages were asymptomatic based on RDT and microscopy, respectively. On the other hand, in the lowland village, the prevalence was $67 / 285(23.5 \%)$ and $13 / 285$ (4.6\%) based on RDT and microscopy, respectively (Table 3 ). It was observed that $133 / 211(63.0 \%)$ of the RDT positive were microscope negative. The proportion of malaria was significantly higher among those aged above 5 years of age (by microscopy and RDT) than those aged below five $(p<0.01)$ (Table 2). P. falciparum was the most predominant malaria parasite detected by RDT and microscopy, $92.5 \%$ and $98.8 \%$, respectively. Plasmodium malariae was detected only in one sample by microscopy. Of the microscopically positive samples, none was infected with

Table 2 Overall prevalence of malaria in the highland and lowland based on malaria rapid diagnostic tests results and microscopy results

\begin{tabular}{|c|c|c|c|c|c|c|}
\hline \multirow[t]{2}{*}{ Variable } & \multicolumn{2}{|l|}{ RDT } & \multirow[t]{2}{*}{ p-value } & \multicolumn{2}{|l|}{ Microscopy } & \multirow[t]{2}{*}{ p-value } \\
\hline & Positive (\%) & Negative (\%) & & Positive (\%) & Negative (\%) & \\
\hline \multicolumn{7}{|l|}{ Age groups } \\
\hline $0-4$ years $(n=237)$ & $62(26.27)$ & $175(73.73)$ & $<0.01$ & $15(6.38)$ & $222(93.62)$ & $<0.01$ \\
\hline $5-9$ years $(n=145)$ & $53(36.55)$ & $92(63.45)$ & & $20(13.79)$ & $125(86.21)$ & \\
\hline $10-14$ years $(n=118)$ & $67(43.2)$ & $51(56.8)$ & & $33(27.97)$ & $85(72.03)$ & \\
\hline $15-19$ years $(n=65)$ & $29(44.6)$ & $36(55.4)$ & & $13(20.00)$ & $52(80.00)$ & \\
\hline \multicolumn{7}{|l|}{ Strata } \\
\hline Highland village $(n=280)$ & $107(38.21)$ & $173(61.79)$ & 0.696 & $52(18.57)$ & $228(81.43)$ & 0.05 \\
\hline Lowland village $(n=285)$ & $104(36.62)$ & $181(63.38)$ & & $29(10.25)$ & $256(89.75)$ & \\
\hline Total $(\mathrm{N}=565)$ & $211(37.41)$ & 354 (62.59) & & 81 (14.39) & $484(85.61)$ & \\
\hline
\end{tabular}

$p<0.01$ the proportion of malaria significantly high in children above five years by both RDT and microscopy and $p=0.05$ the prevalence of malaria in the borderline between the highland and lowland village (in bold) 
Table 3 Asymptomatic malaria infection by age groups and strata as detected by malaria rapid diagnostic test and microscopy

\begin{tabular}{|c|c|c|c|c|c|c|c|}
\hline \multirow[t]{2}{*}{ Variable } & \multirow[t]{2}{*}{$\mathrm{n}$} & \multicolumn{2}{|l|}{ RDT } & \multirow[t]{2}{*}{ p-value } & \multicolumn{2}{|l|}{ Microscopy } & \multirow[t]{2}{*}{$\mathrm{p}$-value } \\
\hline & & Positive (\%) & Negative (\%) & & Positive (\%) & Negative (\%) & \\
\hline \multicolumn{8}{|l|}{ Age groups (years) } \\
\hline $0-4$ & 237 & $40(16.9)$ & $197(83.1)$ & & $6(2.53)$ & $231(97.5)$ & \\
\hline $5-9$ & 145 & $34(23.6)$ & $111(76.6)$ & $<0.01$ & $10(6.9)$ & $135(93.1)$ & $<0.01$ \\
\hline $10-14$ & 118 & $38(32.2)$ & $80(67.8)$ & & $13(11.0)$ & $105(89.0)$ & \\
\hline $15-19$ & 65 & $18(27.7)$ & $47(72.3)$ & & $4(6.2)$ & $61(94.0)$ & \\
\hline \multicolumn{8}{|l|}{ Strata } \\
\hline Highland village & 280 & $63(22.5)$ & $217(77.5)$ & & $20(7.1)$ & $260(93.0)$ & \\
\hline Lowland village & 285 & $67(23.5)$ & $218(76.5)$ & 0.78 & $13(4.6)$ & $272(95.4)$ & 0.19 \\
\hline Total & 565 & $130(23.0)$ & $435(77.0)$ & & $33(5.8)$ & $532(94.2)$ & \\
\hline
\end{tabular}

$p<0.01$ the proportion of asymptomatic malaria significantly high in the age group of 5-9 years by both RDT and microscopy compared to other age groups (in bold)

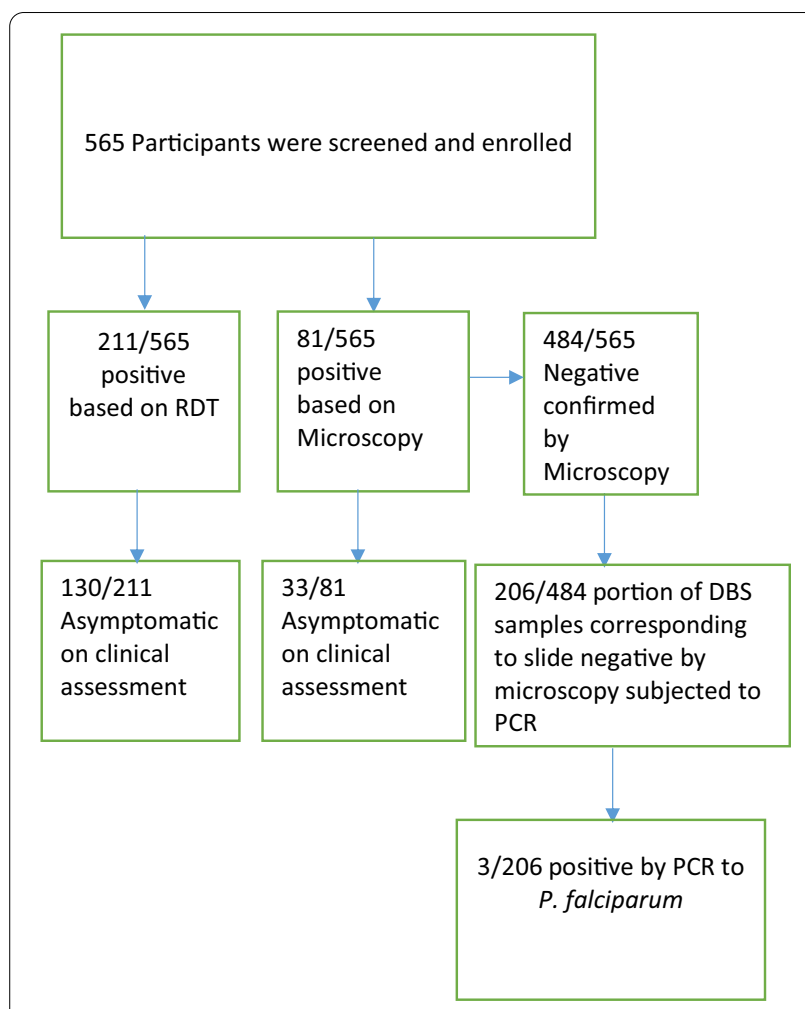

Fig. 1 Consort diagram showing sampling strategies, data collection and downstream analyses

a mixture of Plasmodium species. Only $0.85 \%$ (3/354) of the RDT negatives samples were found slide positive. Plasmodium falciparum gametocytes were detected in $6.2 \%(5 / 81)$ of the samples by microscopy. The prevalence of submicroscopic infection based on PCR was $1.5 \%$ $(3 / 206)$ (Fig. 1). None of the other Plasmodium species ( $P$. malariae and $P$. ovale) were detected by PCR. The results indicated that the majority of the study participants $10.6 \%(60 / 565)$ who mentioned the use of anti-malarial within the past 2 weeks have taken ALU, while one participant have taken sulfadoxine-pyrimethamine.

\section{Prevalence of anaemia}

Of the 565 individuals screened, 135 (23.9\%) were anaemic $(\mathrm{Hb}<11 \mathrm{~g} / \mathrm{dL})$. Among the anaemic cases, 78/135 (57.8\%) had mild anaemia, 50/135 (37.0\%) had moderate anaemia and 7/135 (5.2\%) were severely anaemic. There was no significant difference in the prevalence of anaemia between the low- and highland villages. There was no statistically significant difference in anaemia statuses between males and females $(p=0.25)$. Of the anaemic cases, anaemia seemed to vary with age groups but the difference was not statistically significant $(p=0.22)$ (Table 4).

\section{Association between asymptomatic malaria and anaemia}

The associations between asymptomatic malaria and anaemia $(\mathrm{Hb}<11 \mathrm{~g} / \mathrm{dL})$, gender, age and altitude are explored in Table 5. Model 1 included asymptomatic malaria based on microscopy adjusted for gender, age and altitude. Model 2 included asymptomatic malaria based on RDT adjusted for gender, age and altitude. The risk of anaemia was significantly higher among the participants with asymptomatic malaria based on RDT $(\mathrm{AOR}=2.06$; 95\% CI 1.32-3.20) adjusted for gender, age and altitude. Also based on microscopy results, participants with asymptomatic malaria had risk of anaemia, though not statistically significant (AOR $=2.09 ; 95 \%$ CI $0.98-4.47)$. Males with asymptomatic malaria seemingly had higher 
Table 4 Anaemia status of the study participants by gender, age groups and strata

\begin{tabular}{|c|c|c|c|c|c|c|}
\hline \multirow[t]{2}{*}{ Variable } & \multirow{2}{*}{$\begin{array}{l}\text { Non anaemic } \\
\text { n (\%) }\end{array}$} & \multicolumn{3}{|l|}{ Anaemia } & \multirow{2}{*}{$\begin{array}{l}\text { Total } \\
\mathrm{n}\end{array}$} & \multirow[t]{2}{*}{ P-value } \\
\hline & & $\begin{array}{l}\text { Mild, } \\
\text { n (\%) }\end{array}$ & $\begin{array}{l}\text { Moderate } \\
\text { n (\%) }\end{array}$ & $\begin{array}{l}\text { Severe } \\
\mathrm{n}(\%)\end{array}$ & & \\
\hline \multicolumn{7}{|l|}{ Sex } \\
\hline Male & $199(72.4)$ & $44(16.0)$ & $28(10.1)$ & $4(1.50)$ & $275(48.7)$ & 0.25 \\
\hline Female & $231(79.7)$ & $34(11.7)$ & $22(7.6)$ & $3(1.03)$ & $290(51.3)$ & \\
\hline \multicolumn{7}{|l|}{ Age groups (years) } \\
\hline $0.5-4$ & $174(73.4)$ & $43(18.1)$ & $17(7.2)$ & $3(1.3)$ & $237(42.0)$ & 0.22 \\
\hline $5-11$ & $153(76.9)$ & $19(9.6)$ & $23(11.5)$ & $4(2.0)$ & $199(35.2)$ & \\
\hline $12-14$ & $52(81.3)$ & $7(10.9)$ & $5(7.8)$ & $0(0.0)$ & $64(11.3)$ & \\
\hline $15-19$ & $51(78.5)$ & $9(13.9)$ & $5(7.6)$ & $0(0.0)$ & $61(11.5)$ & \\
\hline \multicolumn{7}{|l|}{ Strata } \\
\hline Highland village & $217(77.5)$ & $38(13.6)$ & $22(7.9)$ & $3(1.0)$ & $280(49.7)$ & 0.82 \\
\hline Lowland village & $213(74.7)$ & $40(14.0)$ & $28(9.8)$ & $4(1.4)$ & $285(50.3)$ & \\
\hline Total & $430(76.1)$ & $78(13.8)$ & $50(8.9)$ & $7(1.2)$ & $565(100)$ & \\
\hline
\end{tabular}

Haemoglobin level $<7.0 \mathrm{~g} / \mathrm{dL}=$ severe anaemia, between 7.0 and $9.9 \mathrm{~g} / \mathrm{dL}=$ moderate anaemia, between 10.0 and $10.9 \mathrm{~g} / \mathrm{dL}=\mathrm{mild}$ anaemia and $\mathrm{Hb} \geq 11 \mathrm{~g} / \mathrm{dL}=\mathrm{non}-$ anaemic. The age groups presented here are as per WHO haemoglobin concentrations for the diagnosis of anaemia and assessment of severity [17]

Table 5 Univariate and multivariate associations of anaemia with asymptomatic malaria using microscopy, RDT, adjusted for age, sex and altitude

\begin{tabular}{|c|c|c|c|c|c|c|}
\hline \multirow[t]{2}{*}{ Variable } & \multirow[t]{2}{*}{ Unadjusted OR $95 \% \mathrm{Cl}$} & \multirow[t]{2}{*}{$p$-values } & Model 1 & P-values & Model 2 & P-values \\
\hline & & & \multicolumn{2}{|l|}{ Adjusted OR, 95\% Cl } & \multicolumn{2}{|l|}{ Adjusted OR, $95 \% \mathrm{Cl}$} \\
\hline \multicolumn{7}{|c|}{ Asymptomatic based on microscopy } \\
\hline Negative & Ref & & Ref & - & - & - \\
\hline Positive & $1.90(0.90-4.0)$ & 0.09 & $2.1(1.0-4.5)$ & 0.56 & - & - \\
\hline \multicolumn{7}{|c|}{ Age group (yr) } \\
\hline$<5$ & Ref & & Ref & & Ref & \\
\hline $5-11$ & $0.8(0.5-1.3)$ & 0.41 & $0.8(0.5-1.2)$ & 0.25 & $0.8(0.5-1.2)$ & 0.21 \\
\hline $12-14$ & $0.6(0.3-1.3)$ & 0.20 & $0.6(0.3-1.2)$ & 0.13 & $0.6(0.3-1.1)$ & 0.11 \\
\hline $15-19$ & $0.8(0.4-1.5)$ & 0.41 & $0.7(0.4-1.5)$ & 0.38 & $0.7(0.4-1.4)$ & 0.30 \\
\hline \multicolumn{7}{|l|}{ Sex } \\
\hline Male & Ref & & Ref & & Ref & \\
\hline Female & $0.7(0.5-1.0)^{*}$ & & $0.7(0.5-1.0)$ & 0.53 & $1.0(0.1-1.0)^{*}$ & 0.04 \\
\hline \multicolumn{7}{|l|}{ Altitude } \\
\hline High & Ref & & Ref & & Ref & \\
\hline Low & $1.2(0.8-1.7)$ & 00.44 & $1.2(0.8-1.8)$ & 0.40 & $1.2(0.8-1.7)$ & 0.49 \\
\hline \multicolumn{7}{|c|}{ Asymptomatic based on RDT } \\
\hline Negative & Ref & - & - & - & Ref & \\
\hline Positive & $1.9(1.3-2.8)^{*}$ & 0.03 & - & - & $2.1(1.3-3.2)^{*}$ & 0.01 \\
\hline
\end{tabular}

* $\mathrm{p}<0.05$, Model 1 included asymptomatic malaria based on microscopic results adjusted for gender, age and altitude whilst model 2 included asymptomatic malaria based on RDT adjusted for gender, age and altitude. The age groups presented here are as per the WHO haemoglobin concentrations for the diagnosis of anaemia and assessment of severity [17]

risk of having anaemia by $34 \%$ when based on RDT (AOR $=1,95 \% \mathrm{CI} 0.45-0.99)$ but was not significantly based on microscopy $(\mathrm{AOR}=0.68 ; 95 \% \mathrm{CI} 0.46-1.01)$. There were no statistically significant differences between having asymptomatic malaria on explanatory variables of altitude and age groups and the risk of having anaemia.

\section{Discussion}

The present study revealed a high prevalence of asymptomatic malaria in this rural population of north-eastern Tanzania. Interestingly, children aged above 5 years who are usually forgotten in many malaria control interventions were particularly vulnerable. It is high time now 
that instead of focusing on underfive and pregnant women, targeted malaria control interventions also consider school aged children who seem to be most vulnerable. Such a high proportion of asymptomatic malaria among those aged above 5 years suggests that school aged children (and above) are a major reservoir of malaria which is in line with other malaria studies from school aged children in Tanzania [18-20]. This raises an alarm for having targeted malaria control programmes for this particular group as these asymptomatic infections may result into persistently low level parasitaemia that may precipitate into anaemia and hence affect cognitive ability among school aged children.

As previously reported [22], the majority of infections were due to $P$. falciparum which is implicated for the more severe form of malaria pathogenesis. However, there were very few infections with $P$. malariae, and a $P$. ovale infection was not detected.

As expected, the study has noted remarkable differences in the detection of malaria by using microscopy and RDT. Notwithstanding, a better picture would have been possible if all RDT positive and all microscopy negative results could be subjected to species diagnostic PCR in order to rule out chances for false positives due to circulating parasite antigens or inability of detection by microscopy for submicroscopic infections. However, upon subjecting a calculated portion of both RDT and microscopy negative samples to malarial species diagnostic PCR, only $1.5 \%$ of the negative samples were confirmed to be sub-microscopic infections that were missed by both RDT and microscopy. Unfortunately, due to limitations of the study we did not have the opportunity to explore all RDT positive/microscopy negative samples by PCR. By PCR the current study analysed a subset of samples that were both negative by RDT and microscopy, this might have an impact on the results for submicroscopic as there were possibility of DBS selected to have false negative results by RDT as results of $P$. falciparum HRP2 gene deletion. The observed percentage of RDT negative which were found slide positive was very low hence chances of having HRP2 gene deleted samples in PCR analyses for submicroscopic was very low as well. Moreover, the current information reported on P. falciparum HRP2 gene deletion in Tanzanian community surveys shows HRP2 gene deletion is uncommon [32].

From this study, it is most likely that RDT may have overestimated the actual malaria point prevalence in the study area as previously reported elsewhere [13, 21, 23, 24]. Apart from being asymptomatic cases, without having PCR or equally sensitive or superior test, it would not be possible to have correct evaluation of the actual point prevalence based on RDT only as some of the positive cases could be false positives and this is one of the weaknesses for the current study.
It is worth mentioning, at least from an epidemiological viewpoint, despite being false positive and having the loophole for overestimating the actual point prevalence, in a resource limited setting like in Korogwe Tanzania, the use of RDT still provides some important information about current/recent infection and could guide attending clinician on what management to offer while ruling out the possibility of having malaria infection at least for those who are RDT negative. The challenge with the HRP-2-based RDTs is the detection of circulating P. falciparum HRP-2 in the blood stream for at least 2 weeks even after successful clearance of infected erythrocytes [25].

The current study observed no significant differences in the prevalence of asymptomatic malaria between the high- and lowland villages as revealed by either RDT and/or microscopy results. This finding is in contrary with a previous observation from the same study area in the period of 2003-2008 [7], however, this may be due to a combination of factors such as climate and environmental changes [26-28].

The overall prevalence of anaemia in this study, defined as $\mathrm{Hb}$ level $<11 \mathrm{~g} / \mathrm{dL}$ was found to be in the category of moderate anaemia (20.0-39.9\%). According to the WHO criteria on assessment for severity of anaemia [17], this finding implies that the study participants in this study area were significantly anaemic and raises some concerns. Individuals from the lowland village were at higher risk of having anaemia as compared to those from the highland, this phenomenon is in agreement with what has been reported previously from the same study sites [7]. Anaemia was associated with the asymptomatic malaria cases as diagnosed by RDT and microscopy which is in line with a previous study from Tanzania [29]. Other factors such as hookworm infestation schistosomiasis and nutritional factors were not assessed and these could have an influence on the observed prevalence of anaemia. With the absence of targeted malaria control efforts among school age children that is usually left out in many malaria control interventions, it is worrisome if this particular group is not included, all malaria control efforts might be highly compromised.

In this study, only few $P$. falciparum gametocytes were observed by microscopy which is contrary to previous studies that have shown a high prevalence of gametocyte carriage in settings with high burden of asymptomatic infections [30]. Therefore, sensitive diagnostic tools for the detection of submicroscopic gametocytes in this setting may be required in order to have a proper guidance for future implementation of strategies aimed at interrupting and curtailing transmission by targeting highrisk transmission hotspots through community-wide 
mass drug administration (MDA), seasonal malaria chemoprevention (SMC), mass screening and treatment campaigns (MSTC) and/or the use of single low dose of primaquine in elimination programmes [31].

\section{Study limitations}

The submicroscopic component of the study analysed DBS samples by PCR that were all RDT negative. This might have an impact on results presented for submicroscopic as there where possibility of false negatives due to $p f h r p 2$ gene deletion.

\section{Conclusions}

In this study, asymptomatic malaria has been shown as a risk factor for having anaemia in the two study communities of north-eastern Tanzania. The findings highlight the need for targeted interventions focusing on asymptomatic infections which sustain the potential for continued transmission of malaria in the study area. Furthermore, the fact that children above 5 years of age were particularly vulnerable to malaria as opposed to under-fives warrants for the introduction and rolling out targeted malaria control interventions focusing on school aged children.

\section{Acknowledgements}

The authors would like to thanks study team and participants for their involvement in the survey. Dr. Bruno Mmbando, Filbert Francis and Daniel Challe are thanked for statistical advice. Christopher Masaka and Lucy Ngowi are thanked for logistical support.

\section{Authors' contributions}

$\mathrm{PMH}$ has been involved in all phases of the study including study design, data collection, analysis, interpretation and wrote the first draft of the manuscript. RAM, PMH and VB did laboratory work. GM, DTRM, EL, MA, CWW, and JPAL participated in the study design and writing the manuscript. All authors read and approved the final vmanuscript.

\section{Funding}

The field component of this study was supported by the European and Developing Countries Clinical Trial Partnership (EDCTP) through the Malaria Research Capacity Building for field Clinical Trials in Tanzania, (MaReCa ProjectTMA 2015 SF-998). The Laboratory work was supported by the same project under MSc study scholarship for Paul Martine Hayuma.

\section{Availability of data and materials}

The dataset generated and/or analysed during the current study are available from the corresponding author on reasonable request.

\section{Declarations}

The finding and conclusion presented in this report are those of the authors based on the data presented which was part of the main ongoing MaReCa Project in north-eastern Tanzania.

\section{Ethics approval and consent to participate}

The study received ethical approval certificate from the Medical Research Coordinating Committee of the National Institute for Medical Research (NIMRMRCC), Tanzania, with reference number NIMR/HQ/R.8a/Nol.IX/2804. The study was also approved by Institutional Review Board (IRB) of Sokoine University of Agriculture (SUA) with reference number SUA/ADM/R.1/8/232. Prior to data collection, sensitization meetings were held with potential study participants in the respective study communities. The study objectives were explained to participants in Kiswahili, a language understood by the parents and guardians with emphasis laid upon voluntary participation. Written informed consent to participate was obtained from each study participant or their parents/ guardians.

\section{Consent for publication}

This manuscript is published with permission from the Director General of the National Institute for Medical Research, Tanzania.

\section{Competing interests}

The authors declare that they have no competing interests.

\section{Author details}

${ }^{1}$ National Institute for Medical Research, Tanga Research Centre, P. O. Box 5004, Tanga, Tanzania. ${ }^{2}$ Department of Veterinary Microbiology, Parasitology and Biotechnology, College of Veterinary Medicine and Biomedical Sciences, Sokoine University of Agriculture, P. O. Box 3019, Morogoro, Tanzania. ${ }^{3}$ Department of Immunology and Microbiology, Centre for Medical Parasitology, University of Copenhagen, Copenhagen, Denmark. ${ }^{4}$ Department of Infectious Diseases, Copenhagen University Hospital, Copenhagen, Denmark. ${ }^{5}$ SACIDS Africa Centre of Excellence for Infectious Diseases, SACIDS Foundation for One Health, Sokoine University of Agriculture, P. O. Box 3297, Morogoro, Tanzania.

Received: 29 March 2021 Accepted: 13 October 2021

Published online: 29 October 2021

\section{References}

1. WHO. World malaria report 2020: 20 years of global progress and challenges. Geneva: World Health Organization. 2020. https://www.who.int/ publications/i/item/9789240015791. Accessed 01 Jan 2021

2. Bhatt S, Weiss DJ, Cameron E, Bisanzio D, Mappin B, Dalrymple U, et al. The effect of malaria control on Plasmodium falciparum in Africa between 2000 and 2015. Nature. 2015;526:207-11.

3. Tanzania National Malaria Control Programme. Malaria surveillance bulletin 2018. Dar es Salaam. https://www.measureevaluation.org/measu re-evaluation-tz/malaria/Malaria\%20Bulletin\%20issue\%203.pdf. Accessed 20 July 2020

4. Mahende C, Ngasala B, Lusingu J, Yong T-S, Lushino P, Lemnge M, et al. Performance of rapid diagnostic test, blood-film microscopy and PCR for the diagnosis of malaria infection among febrile children from Korogwe District, Tanzania. Malar J. 2016:15:391.

5. Drakeley C, Abdulla S, Agnandji ST, Fernandes JF, Kremsner P, Lell B, et al. Longitudinal estimation of Plasmodium falciparum prevalence in relation to malaria prevention measures in six sub-Saharan African countries. Malar J. 2017;16:433.

6. Ishengoma DS, Mmbando BP, Segeja MD, Alifrangis M, Lemnge MM, Bygbjerg IC. Declining burden of malaria over two decades in a rural community of Muheza district, north-eastern Tanzania. Malar J. 2013;12:388.

7. Mmbando BP, Vestergaard LS, Kitua AY, Lemnge MM, Theander TG, Lusingu JPA. A progressive declining in the burden of malaria in northeastern Tanzania. Malar J. 2010;9:216.

8. Rek J, Katrak S, Obasi H, Nayebare P, Katureebe A, Kakande E, et al. Characterizing microscopic and submicroscopic malaria parasitaemia at three sites with varied transmission intensity in Uganda. Malar J. 2016;15:470.

9. Roper C, Elhassan IM, Hviid L, Giha H, Richardson W, Babiker H, et al. Detection of very low level Plasmodium falciparum infections using the nested polymerase chain reaction and a reassessment of the epidemiology of unstable malaria in Sudan. Am J Trop Med Hyg. 1996;54:325-31.

10. Lusingu JPA, Vestergaard LS, Mmbando BP, Drakeley CJ, Jones C, Akida J, et al. Malaria morbidity and immunity among residents of villages with different Plasmodium falciparum transmission intensity in north-eastern Tanzania. Malar J. 2004;3:26.

11. Gudo ES, Prista A, Jani IV. Impact of asymptomatic Plasmodium falciparum parasitemia on the imunohematological indices among school children and adolescents in a rural area highly endemic for malaria in southern Mozambique. BMC Infect Dis. 2013;13:244. 
12. Jones R, Roschnik N, Jukes MC, Clarke SE, Diarra S, Traore K, et al. Impact of a malaria intervention package in schools on Plasmodium infection, anaemia and cognitive function in schoolchildren in Mali: a pragmatic cluster-randomised trial. BMJ Glob Health. 2017;2:e000182.

13. Lee JH, Jang JW, Cho CH, Kim JY, Han ET, Yun SG, et al. False-positive results for rapid diagnostic tests for malaria in patients with rheumatoid factor. J Clin Microbiol. 2014;52:3784-7.

14. Ministry of Health and Social Welfare. National guidelines for diagnosis and treatment of malaria in Tanzania. Dar es Salaam; 2013. http://apps. who.int/medicinedocs/documents/s19271en/s19271en.pdf.

15. Singh B, Bobogare A, Cox-Singh J, Snounou G, Abdullah MS, Rahman HA. A genus- and species-specific nested polymerase chain reaction malaria detection assay for epidemiologic studies. Am J Trop Med Hyg. 1999;60:687-92.

16. Bousema T, Okell L, Felger I, Drakeley C. Asymptomatic malaria infections: detectability, transmissibility and public health relevance. Nat Rev Microbiol. 2014;12:833-40.

17. WHO. Haemoglobin concentrations for the diagnosis of anaemia and assessment of severity. Geneva: World Health Organization; 2011. https:// www.who.int/vmnis/indicators/haemoglobin/en/.

18. Mboera LEG, Bwana VM, Rumisha SF, Malima RC, Mlozi MRS, Mayala BK, et al. Malaria, anaemia and nutritional status among schoolchildren in relation to ecosystems, livelihoods and health systems in Kilosa District in central Tanzania global health. BMC Public Health. 2015;15:553.

19. Nzobo BJ, Ngasala BE, Kihamia CM. Prevalence of asymptomatic malaria infection and use of different malaria control measures among primary school children in Morogoro Municipality, Tanzania. Malar J. 2015;14:491.

20. Chacky F, Runge M, Rumisha SF, Machafuko P, Chaki P, Massaga JJ, et al. Nationwide school malaria parasitaemia survey in public primary schools, the United Republic of Tanzania. Malar J. 2018;17:452.

21. Bell DR, Martin LB, Wilson DW. False-positive results of a Plasmodium falciparum histidine-rich protein 2-detecting malaria rapid diagnostic test due to high sensitivity in a community with fluctuating low parasite density. Am J Trop Med Hyg. 2018;73:199-203.

22. Kim MJ, Jung BK, Chai JY, Eom KS, Yong TS, Min DY, et al. High malaria prevalence among schoolchildren on Kome Island, Tanzania. Korean J Parasitol. 2015;53:571-4.

23. Humar A, Ohrt C, Harrington MA, Pillai D, Kain KC. ParaSight ${ }^{\circledR}$ F test compared with the polymerase chain reaction and microscopy for the diagnosis of Plasmodium falciparum malaria in travelers. Am J Trop Med Hyg. 1998;92:166-9.

24. Keating J, Miller JM, Bennett A, Moonga HB, Eisele TP. Plasmodium falciparum parasite infection prevalence from a household survey in Zambia using microscopy and a rapid diagnostic test: implications for monitoring and evaluation. Acta Trop. 2009;112:277-82.

25. Harris I, Sharrock W, Bain L, Gray KA, Bobogare A, Lilley K, et al. A large proportion of asymptomatic malaria infections with low parasite densities in Temotu Province, Solomon Islands: challenges for malaria diagnostics in an elimination setting. Malar J. 2010;9:254.

26. Yanda PZ, Kangalawe RY, Sigalla RJ. Climatic and socio-economic influences on malaria and cholera risks in the Lake Victoria region of Tanzania. AIACC Working Papers; 2005.

27. Himeidan YE-S, Kweka E. Malaria in East African highlands during the past 30 years: impact of environmental changes. Front Physiol. 2012;3:315.

28. Wandiga SO, Opondo M, Olago D, Githeko A, Githui F, Marshall M, et al. Vulnerability to epidemic malaria in the highlands of Lake Victoria basin: the role of climate change/variability, hydrology and socio-economic factors. Clim Change. 2010;99:473-97.

29. Schellenberg D, Schellenberg JRMA, Mushi A, De Savigny D, Mgalula L, Mbuya C, et al. The silent burden of anaemia in Tanzanian children: a community-based study. Bull World Health Organ. 2003;81:581-90.

30. Vantaux A, Samreth R, Piv E, Khim N, Kim S, Berne L, et al. Contribution to malaria transmission of symptomatic and asymptomatic parasite carriers in Cambodia. J Infect Dis. 2018;217:1561-8.

31. Nankabirwa JI, Wandera B, Amuge P, Kiwanuka N, Dorsey G, Rosenthal PJ, et al. Impact of intermittent preventive treatment with dihydroartemisinin-piperaquine on malaria in Ugandan schoolchildren: a randomized, placebo-controlled trial. Clin Infect Dis. 2014;58:1404-12.

32. Bakari C, Jones S, Subramaniam G, Mandara Cl, Chiduo MG, Rumisha S, et al. Community-based surveys for Plasmodium falciparum pfhrp2 and pfhrp3 gene deletions in selected regions of mainland Tanzania. Malar J. 2020;19:391.

\section{Publisher's Note}

Springer Nature remains neutral with regard to jurisdictional claims in published maps and institutional affiliations.
Ready to submit your research? Choose BMC and benefit from:

- fast, convenient online submission

- thorough peer review by experienced researchers in your field

- rapid publication on acceptance

- support for research data, including large and complex data types

- gold Open Access which fosters wider collaboration and increased citations

- maximum visibility for your research: over $100 \mathrm{M}$ website views per year

At BMC, research is always in progress.

Learn more biomedcentral.com/submissions 